\title{
Ein methodischer Blick auf Tarif und Tarifanpassung
}

\author{
Andreas Bührer \\ Dr. sc. nat. et med., Andreas Bührer Consulting, Weingarten-Kalthäusern
}

Die gesamtschweizerisch geltende Tarifstruktur TARMED basiert auf dem Tarifmodell TARMED. Die Preisbildung erfolgt durch die Verhandlung des Taxpunktwerts für jede Vertragsgemeinschaft einzeln. Die Gesamtkosten im KVG ergeben sich aus «Menge» mal "Preis». Daraus folgt, dass die Gesamtkosten nicht allein über die Tarifstruktur und die Tarife gesteuert werden können.

\begin{abstract}
Das Tarifmodell TARMED garantiert die betriebswirtschaftliche Bemessung.

Das Tarifmodell umfasst das betriebswirtschaftliche Denkmodell und die daraus abgeleiteten Berechnungsalgorithmen. Generell haben Modelle die Aufgabe, die Realität möglichst wirklichkeitsnah abzubilden.
\end{abstract}

\section{Die Tarifstruktur ist das Resultat der} Anwendung des Tarifmodells.

Die Tarifstruktur ist das Resultat der Anwendung des Tarifmodells. Sie setzt sich aus der Nomenklatur, der positionsbezogenen Leistungsbewertung und der Anzahl Taxpunkte für die ärztliche Leistung AL und die technische Leistung TL zusammen. Dabei entspricht ein Taxpunkt einem Schweizer Franken.

\section{Die Tarifpflege ist wichtig.}

Die Tarifstruktur muss regelmässig revidiert und gepflegt werden. Sonst veraltet sie und verliert ihre Sachgerechtigkeit. Die Tarifstruktur TARMED wurde seit ihrer Einführung vor über zehn Jahren noch nie umfassend revidiert. Deshalb ist die Entwicklung der Kosten in der aktuellen Tarifstruktur (1.08.00_BR) noch nicht nachvollzogen. Es ist davon auszugehen, dass die betriebswirtschaftliche Bemessung einer neuen Tarifstruktur zu höheren Taxpunkten führen wird; dies nicht zuletzt aufgrund der Lohnentwicklung des nichtärztlichen Personals.

Eingriffe in die Tarifstruktur haben aus methodischer Sicht einen willkürlichen Charakter. Eingriffe direkt in die Tarifstruktur haben aus streng methodischer Sicht einen willkürlichen Charakter, weil die betriebswirtschaftlichen, im Tarifmodell TAR-
MED berücksichtigten Abhängigkeiten, Regeln und Vereinbarungen ausser Acht gelassen werden. Diese gewährleisten eine betriebswirtschaftlich bemessene und sachgerechte Tarifstruktur.

\section{Die Taxpunktwerte fallen in Richtung Krankenversicherung und Arztpraxen.}

Die zur Anwendung kommenden Taxpunktwerte (so weit diese öffentlich gemacht wurden) variieren zwischen 0.75 CHF und 1.00 CHF. Es zeigt sich dabei, dass gleiche Leistungen durch die Krankenversicherung deutlich schlechter vergütet werden als durch die Unfallversicherung und Arztpraxen schlechter gestellt sind als Spitäler.

Die Gesamtkosten können nicht allein über die Tarifstruktur und die Tarife gesteuert werden, auch wenn das Wirtschaftlichkeitsgebot eingehalten wird.

Die Gesamtkosten sind in der Krankenversicherung ein zentrales Thema. Die Kosten ergeben sich aus «Menge» mal Taxpunktwert mal Taxpunkte. Dabei ist die «Menge» der wichtigste Faktor. Es würde zu kurz greifen, die Mengenzunahme lediglich als ökonomisch getriebene Mengenausweitung zu verstehen. Die Einflussfaktoren auf die «Menge» sind sehr vielschichtig. Faktoren sind zum Beispiel die Anspruchshaltung der Bevölkerung, Einfluss von Medien und Werbung, Qualität, Reglementierung, Gesamtprozessverantwortung oder Fragen zum Umgang mit dem Sterben und dem Tod. Daraus folgt, dass die «Menge» und damit die Gesamtkosten kaum über die Tarifstruktur und die Tarife gesteuert werden können, auch wenn das Wirtschaftlichkeitsgebot maximal eingehalten wird. 
Ein Globalbudget ist für den ambulanten Bereich im KVG nicht vorgesehen.

Ein Globalbudget ist für den ambulanten Bereich im KVG - auch unter den ausserordentlichen Massnahmen zur Eindämmung der Kosten [1] - nicht vorgesehen. Würde ein Globalbudget eingeführt werden wollen, fehlten heute konkrete Ansätze, Vorstellungen und Erfahrungen, wie die Geldmittel für ganze Bevölkerungsgruppen aus medizinischer Sicht ethisch eingesetzt und verteilt werden könnten. Eine Taxpunktwertreduktion als Steuermittel, falls das Budget überschritten würde, wäre keine tragende Lösung und nicht sachgerecht.

\section{Kostenneutralität gilt nur bei Modellwechsel.}

Von einem Globalbudget zu unterscheiden ist die «Kostenneutralität bei Modellwechsel» [2]. Diese bedeutet aus methodischer Sicht, dass die in einem bestimmten Zeitraum, z.B. einem Jahr, erbrachten KVG-Leistungen für die Bestimmung der Vergütung sowohl mit dem alten als auch dem neuen Modell abgebildet werden und dass dabei die Gesamtkosten gleich bleiben. Die kostenneutrale Überführung enthält keine Vorgaben für die zukünftige Kostenentwicklung. Je mehr prospektive Elemente hinzukommen, desto eher handelt es sich um ein verdecktes Globalbudget. Aus methodischer Sicht ist zudem zu fordern, dass das alte Modell revidiert werden muss, bevor die Überführung ins neue Modell erfolgen kann. Sonst wird mit den beiden Modellen nicht die gleiche Realität abgebildet. Wie sollte zudem die alte, nicht mehr sachgerechte Tarifstruktur - weil nicht nachgeführt - gleichzeitig die sachgerechte Basis für die neue Tarifstruktur sein?

\section{Die Forderung nach Kostenneutralität bei} Tarifrevisionen stellt die betriebswirtschaftliche Bemessung und damit die Sachgerechtigkeit in Frage.

Von der Kostenneutralität bei Modellwechsel sind Tarifrevisionen zu unterscheiden. Bei einer Revision des Tarifs ist aus methodischer Sicht keine Kostenneutralität möglich, weil sonst die betriebswirtschaftliche Bemessung, die durch das Tarifmodell gewährleistet wird, verletzt würde.

\section{Die doppelte Kostenneutralität hat die Eigenschaften eines Globalbudgets.}

Obwohl bei einer Tarifrevision aus methodischer Sicht keine Kostenneutralität gelten kann, wird diese oft gefordert und als statische Kostenneutralität bezeichnet. Zusätzlich wird zudem eine dynamische Kostenneutralität gefordert, welche die Entwicklung des Kostenvolumens von Jahr zu Jahr einschränkt. Eine solche doppelte Kostenneutralität hat die Eigenschaften eines Globalbudgets, allerdings ohne dass die Tarifbehörden die Verantwortung für die Zuweisung der Geldmittel übernehmen.

\section{Referenzen}

1 Nach Art. 54, 55, 55a KVG.

2 Nach Art. 59c Abs. 1 lit. c KVV. 